\title{
Reavaliação dos Perfis de Vagarosidades Sônicas no Reservatório Namorado, Bacia de Campos
}

Mário Martins Ramos, Jorge Leonardo Martins, Observatório Nacional (ON)

Copyright 2016, SBGf - Sociedade Brasileira de Geofísica

Este texto foi preparado para a apresentação no VII Simpósio Brasileiro de Geofísica Ouro Preto, 25 a 27 de outubro de 2016. Seu conteúdo foi revisado pelo Comitê Ouro Preto, 25 a 27 de outubro de 2016. Seu conteúdo foi revisado pelo Comitê de seus associados. É proibida a reprodução total ou parcial deste material para propósitos comerciais sem prévia autorização da SBGf.

\begin{abstract}
In the log interpretation, sometimes, its necessary the usage of some models to complete well log intervals whith no data. A lot of them (especially empirical models) are used in the gás/oil industry that are, usually, mathematical models with a linear system solution. These models, generally, are made for a especific enviroment, whith means, that their main applicability are restrict for especific conditions. There are also petrophysical models, that are originated by algebrical manipulations of preexisting equations and physical properties, that result in equations chiefly limited by acquisition methods. The main objective of this work is; to compare this two kinds of models that predict the sonic log, using real data from the Campos basin, specifically, in the Namorado oil field that comprise 38 wells located in Namorado formation, turbidite sandstones lenses that extend from the Cenomanian to Albian. We use several comparison methods in wells that had complete, or partially complete, sonic logs, that were also used to create three maps: acustic impedance, refletivity and seismic amplitude, whose use is common in the reservoir analysis.
\end{abstract}

\section{Introdução}

O perfil sônico mede propriedades acústicas da formação através da relação entre propriedades acústicas e elásticas, o que lhe caracteriza como uma importante ferramenta para a modelagem, avaliação de reservatórios e correlação sismoestratigráfica. No entanto, em diversas situações, estes perfis se encontram incompletos ou ausentes, o que leva a utilização de modelos empíricos e modelos petrofísicos para suprir tais condições. Neste trabalho, nós reavaliamos os modelos empíricos, construídos a partir da regressões por mínimos quadrados, e um modelo petrofísico que utiliza a argilosidade, a porosidade do folhelho e a porosidade efetiva para determinar a vagarosidade, com o objetivo de verificar qual a diferença entre os valores gerados pelos modelos e os valores reais de vagarosidade nos poços; como essa diferença afeta os valores de impedância acústica, refletividade e amplitude sísmica; assim como a interferência da aplicação destes modelos afeta a interpolação dos mapas de impedância acústica, refletividade e amplitude sísmica em uma bacia. Esta avaliação foi realizada no campo de namorado (bacia de Campos), no intervalo de 2940 a 3300 metros de profundidade e que compreende 0 albiano e $o$ cenomaniano da bacia, onde foram utilizados 38 poços, sendo que, destes 38,8 possuem o perfil sônico completo, 2 possuem o sônico incompleto e, nos demais 28 , este perfil esta ausente. Para verificar a diferença entre os valores gerados pelos modelos e os valores reais de vagarosidade nos poços, foram aplicados (onde os perfis estavam completos) ambos os modelos, empíricos e petrofísico que foram avaliados estatisticamente através da correlação e da distribuição dos valores. Afim de entender como a interferência da aplicação dos modelos afeta a interpolação dos mapas, estes modelos foram utilizados também nos demais 30 poços. Em todos os poços, foram calculados os valores de impedância acústica, refletividade e amplitude sísmica, que resultou no banco de dados que foi utilizado para gerar os respectivos mapas utilizando a krigagem simples como interpolador através de um software em linguagem Python que foi elaborado durante trabalho.

\section{Metodologia/ Problema Investigado}

Os dados relativos a estes poços contém 5 perfis cada: sônico, raios gama, resistividade, porosidade neutrônica e densidade, sendo que muitos destes encontram-se incompletos ou ausentes. O procedimento efetuado para cada um dos poços, consiste em calcular os valores dos perfis incompletos ou inexistentes através da regressão por mínimos quadrados, usando relações empíricas descritas por Augusto (2009), para se obter um banco de dados completo, que foi utilizado no cálculo dos modelos.

À partir dos valores do perfil de raios gama, foi calculada a argilosidade $\left(\mathrm{V}_{\mathrm{i}, \mathrm{SH}}\right)$ através da equação empírica de Larionov para sedimentos recentes $\mathrm{V}_{\mathrm{i}, \mathrm{SH}}=0.083\left(2^{3.70 \times}\right.$ ${ }^{1 G R i-1}$ ), onde IGR ${ }^{\mathrm{i}}$ é o valor do índice de raios gama, que é calculado segundo a equação $I G R_{i}=\left(G_{i}-\right.$ $\left.G R_{\min }\right) /\left(G R_{\max }\right.$ - $\left.G R_{\min }\right)$, que considera os valores máximos, mínimos e o i-ésimo valor do perfil de raios gama (respectivamente $\mathrm{GR}_{\max }, \mathrm{GR}_{\min }$ e $\mathrm{GR}_{\mathrm{i}}$ ).

Em seguida, o modelo petrofísico (segundo Siqueira and Martins, 2011) é definido por:

$$
\begin{gathered}
\Delta t_{i, D}=\Delta t_{m a}+\left(\Delta t_{f}-\Delta t_{m a}\right) \times\left[\frac{\rho_{i}-\rho_{m a}}{\rho_{f}-\rho_{m a}}\right. \\
\left.-V_{i, s h}\left(\frac{\rho_{s h}-\rho_{m a}}{\rho_{f}-\rho_{m a}}\right)+V_{i, s h}\left(\frac{\Delta t_{s h}-\Delta t_{m a}}{\Delta t_{f}-\Delta t_{m a}}\right)\right]
\end{gathered}
$$

onde $\rho_{\text {ma }}, \rho_{f}$, e $\rho_{\text {sh }}$ são, respectivamente, as densidades da matriz, do fluído contido na porosidade e do folhelho adjacente, assim como $\Delta t_{m a}, \Delta t_{f}, e \Delta t_{p}$, sh corresponde às vagarosidade da matriz, do fluído contido na porosidade e do folhelho adjacente, sendo $\rho_{\mathrm{i}}$ o i-ésimo valor do perfil de densidade. A densidade do folhelho $\left(\rho_{\text {sh }}\right)$ é calculada à 
partir da porosidade total $\varphi_{\mathrm{i}, \mathrm{t}, \mathrm{D}}$ e da porosidade neutrônica $\varphi_{\mathrm{i}, \mathrm{N}}$, de modo que, o valor máximo da diferença entre estas porosidades $\left(\max \left(\varphi_{\mathrm{i}, \mathrm{N}}-\varphi_{\mathrm{i}, \mathrm{t}, \mathrm{D}))}\right)\right.$ determina $\mathrm{o}$ ponto de folhelho $P$ entre os demais i elementos, e que possui o valor da porosidade do folhelho e é utilizado para determinar $\rho_{\mathrm{P}, \mathrm{sh}}$. Já o valor de $\Delta \mathrm{t}_{\mathrm{sh}}$ é calculado à partir da relação empírica (adaptada de Gardner et al., 1974) VP,sh $=0.1089 \times \rho^{4} \mathrm{P}, \mathrm{sh}$, onde VP,sh é convertido para o valor de $\Delta \mathrm{t}_{\text {sh }}$.

Ambos os modelos são calculados para cada poço, sendo que, os modelos empíricos, possuem uma equação distinta para cada poço, calculada através da regressão por mínimos quadrados, onde os perfis estão completos ou incompletos, e no caso de perfis sônicos ausentes, utilizam a mesma equação do poço mais próximo (Augusto, 2009).

O sismograma sintético $\mathrm{S}(\mathrm{t})$ utilizado é gerado à partir do modelo convolucional

$$
S(t)=w(t) * R(t)+e(t)
$$

onde $\mathrm{w}(\mathrm{t})$ é a wavlet, $\mathrm{R}(\mathrm{t})$ é o contraste de impedância acústica (ou refletividade) e e(t) é o erro associado. Utilizando os valores de vagarosidade, foram obtidas as velocidades de ambos os modelos $V_{E}$ e VPP relativas, respectivamente, aos modelos empíricos e petrofísico, que juntamente com a velocidade original medida nos perfis (VP), foram utilizadas nos cálculos da impedância acústica e da refletividade, que são obtidas, respectivamente, através das equações:

$$
Z_{i}=\rho_{i} \cdot V P_{i}, \text { e } R_{i}=\left(Z_{i}-1-Z\right)_{i} /\left(Z_{i}-1+Z_{i}\right) \text {, }
$$

que foram determinadas para cada modelo, de modo que, a partir das velocidades $V P, V_{E}$ e $V P_{P}$ existem valores de impedância $\left(Z, Z_{E}\right.$ e $\left.Z_{P}\right)$ e de refletividade $(R$, $R_{E}, R_{P}$ ) respectivos ao modelo empírico (sub índice $E$ ) e petrofísico (sub índice P). Neste trabalho, optamos por utilizar a wavlet de Richer

$$
w(t)=A\left(1-2 \pi^{2} 2 f^{2} 2 t^{2}\right)\left[\exp \left(-\pi^{2} f^{2} t^{2}\right)\right],
$$

em que A é a amplitude, f é a frequência e t é o tempo. Esta equação foi aplicada no poço com uma escala regular de tempo (Yilmaz, 1987) calculada à partir dos valores de velocidades e dos intervalos de profundidade, com frequeência de $600 \mathrm{~Hz}$ e A igual a 1, de modo 2 que, dependendo da existência ou não dos perfis sônicos, foram calculadas, também, as amplitudes dervidas do perfil e dos modelos ( $S, S_{E}$ e $S_{P}$ ) apenas para efeito comparativo.

Para criar os mapas de impedância acústica, refletividade e amplitude sísmica, foram utilizadas as propriedades homônimas calculadas em cada poço em na profundiade de 2974, 3038 e 3103m que representam intervalos importantes na distribuição dos reservatórios. Para cada uma destas profundidades, foram utilizados os valores derivados do perfil sônico (aonde este existia), e, nas demais, foram utilizados os valores gerados pelos modelos, de modo a criar dois mapas distintos, para uma mesma profundidade, um com valores derivados do modelo empírico e outro do petrofísico. Utilizamos a krigagem simples como interpolador conforme descrito por Isaaks and Srivastava (1989), cujo variograma foi feito com valores categorizados devido a malha irregular (Sarma 2009). Os mapas foram computados em uma matriz de $500 \times 400$, que representa a região original do mapa que tem $4 \times 5 \mathrm{~km}$, onde cada ponto da matriz equivale a uma área de $100 \mathrm{~m}^{2}(10 \times 10 \mathrm{~m})$.

\section{Resultados}

Inicialmente, foram atribuídos valores tabelados para as constantes nos poços $\left(\rho_{\mathrm{ma}}=2.65, \Delta \mathrm{t}_{\mathrm{ma}}=55.5, \rho_{\mathrm{f}}=1.101\right.$ e $\Delta t_{f}=189.0$ ) retirados de tabela de Gardner et al., 1974, e Magalhães e Martins 2012, que foram aplicados no modelo petrofísico. Utilizando como exemplo o poço NA04 , os resultados da figura 1 demonstram que, mesmo sem ser calibrado com valores do perfil sônico, O perfil sintético apresenta resultados satisfatórios.
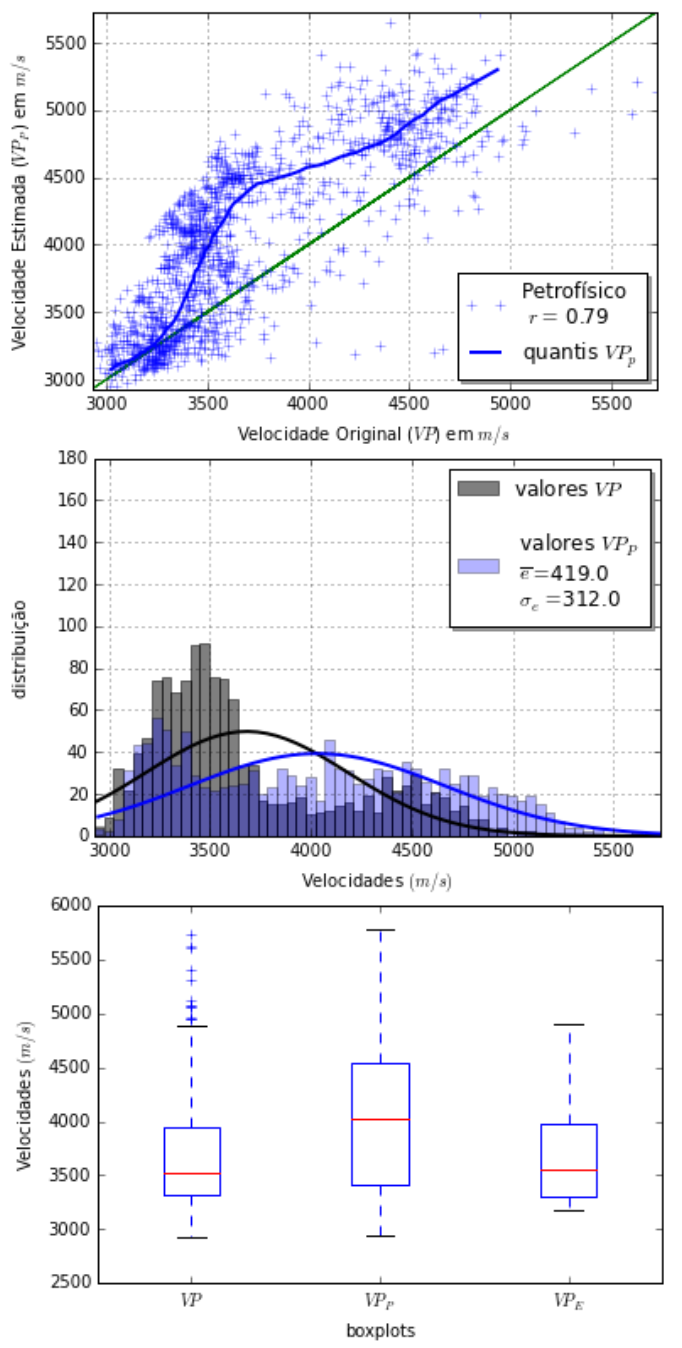

Figura 1 - Gráficos de dispersão, histogramas e diagramas de caixa do modelo petrofísico $\left(V P_{P}\right)$ para o poço NA-04

(observação: $r$ = coeficiente de correlação, $|\mathrm{e}|, \mathrm{e}=$ média do erro, $\sigma_{e}=$ desvio padrão do erro). 
Por questões comparativas, executamos as mesmas análises que foram atribuídas na determinação de VPP em VPE (figura 2) que apresentaram melhores resultados em relação ao modelo petrofísico. É importante salientar que o modelo empírico utilizado na comparação foi justamente aquele que obteve melhores resultados, que foi determinada através da regressão por mínimos quadrados, utilizando (inclusive) valores VP originários do poço para calibração. Quanto ao gráfico de dispersão e aos valores dos quantis, é evidente o melhor ajuste promovido pelo modelo empírico, ajuste que é também observado nos diagramas de caixa. Já no histograma e na curva gaussiana, os resultados não foram tão favoráveis ao modelo de $\mathrm{VP}_{\mathrm{E}}$.
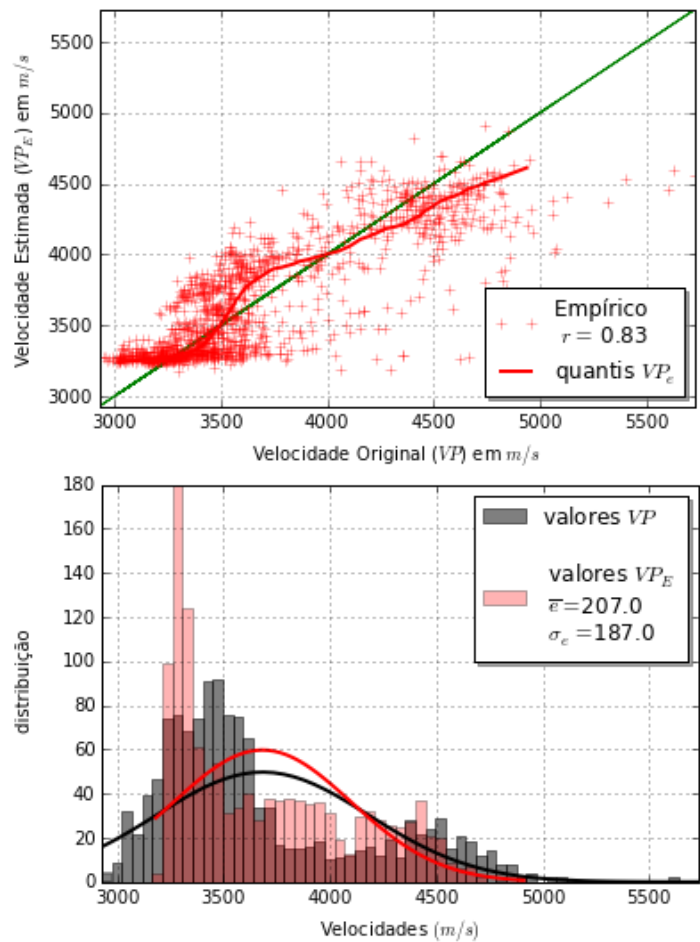

Figura 2 - Gráficos de dispersão e histogramas do modelo petrofísico $\left(V P_{E}\right)$ para o poço NA-04

Testamos também um segundo modelo (VP $\mathrm{P}_{\mathrm{P}, \mathrm{M}}$ ) cujas constantes de $\rho_{\mathrm{ma}}$ e $\Delta \mathrm{t}_{\mathrm{ma}}$ foram obtidos ao testar diversos valores no modelo petrofísico que, comparados com aqueles obtidos em VP produziram o menor erro. A média destes valores no poço NA-04 foi de 2.5427 para $\rho_{\text {ma }}$ e 70.2399 para $\Delta t_{\text {ma }}$ e, após aplicados ao modelo petrofísico, resultaram nos dados da figura 3. O gráfico de dispersão evidencia que os valores de $V_{P}, M$ se ajustam melhor aos valores de VP devido a curva verde centralizada. A curva referente aos quantis de VP indica um melhor ajuste para velocidades baixas, o que corresponde a maior parte dos dados. Quanto ao

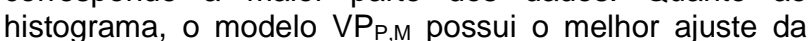
curva gaussiana, característica esta observada em todos os poços.
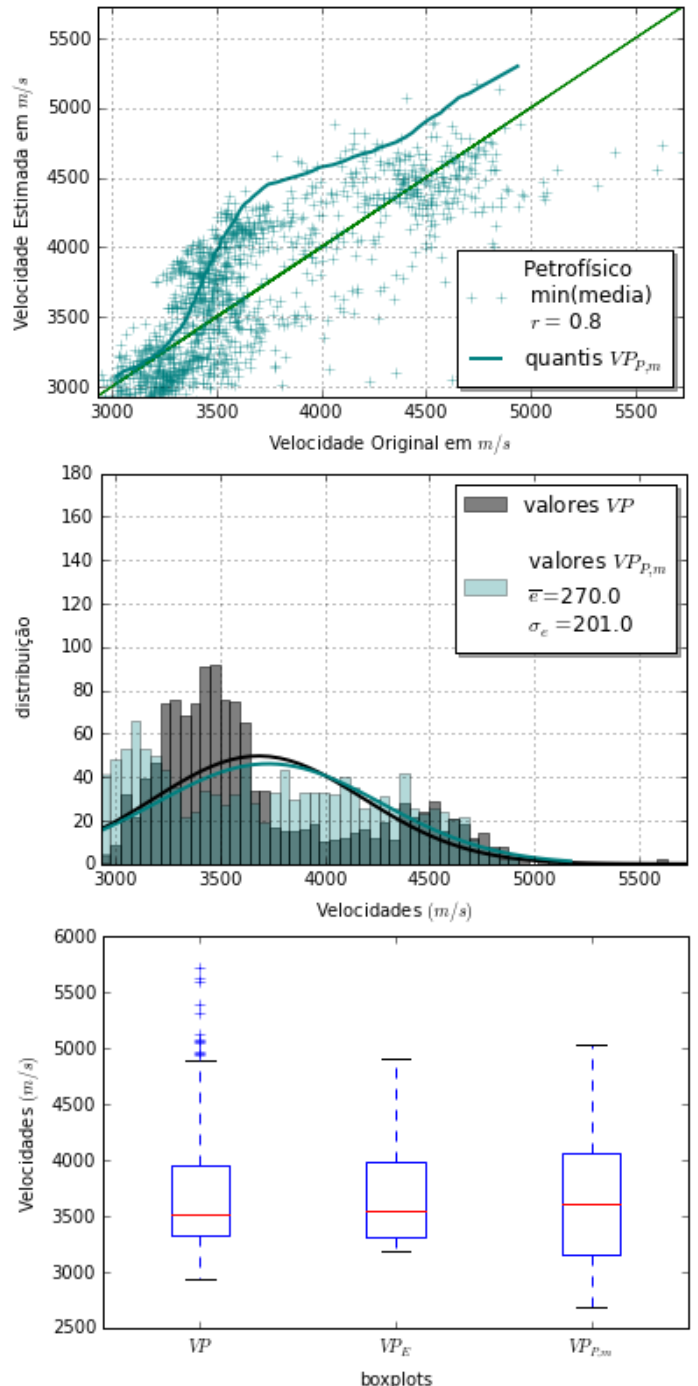

Figura 3 - Gráficos de dispersão, histogramas e diagramas de caixa do modelo petrofísico $\left(V P_{p, m}\right)$ para o poço NA-04

Criamos um modelo que considerasse também a litologia do meio $\left(\mathrm{VP}_{\mathrm{P}, \text { lito }}\right)$, atribuindo valores de $\sigma_{\mathrm{ma}}$ e $\Delta \mathrm{t}_{\mathrm{ma}}$ para as 5 litologias do intervalo, que foram obtidos de modo similar ao modelo VP $P_{P, M}$, onde a média dos valores testados fosse obtida para cada litologia. Este modelo final produziu os melhores resultados dos modelos petrofísicos, o que pode ser observado na figura 4. Estes resultados são melhores pois a categorização segundo a litologia reduz a homogeneização ao se executarem 5 médias (uma para cada litologia) ao invés de 1 para todo o poço. Evidentemente que, se os valores atribuídos às litologias forem utilizados em outros poços, os resultados não serão tão precisos. Este teste foi executado também com valores tabelados e, produziram resultados melhores se comparados aos valores obtidos em VP. 

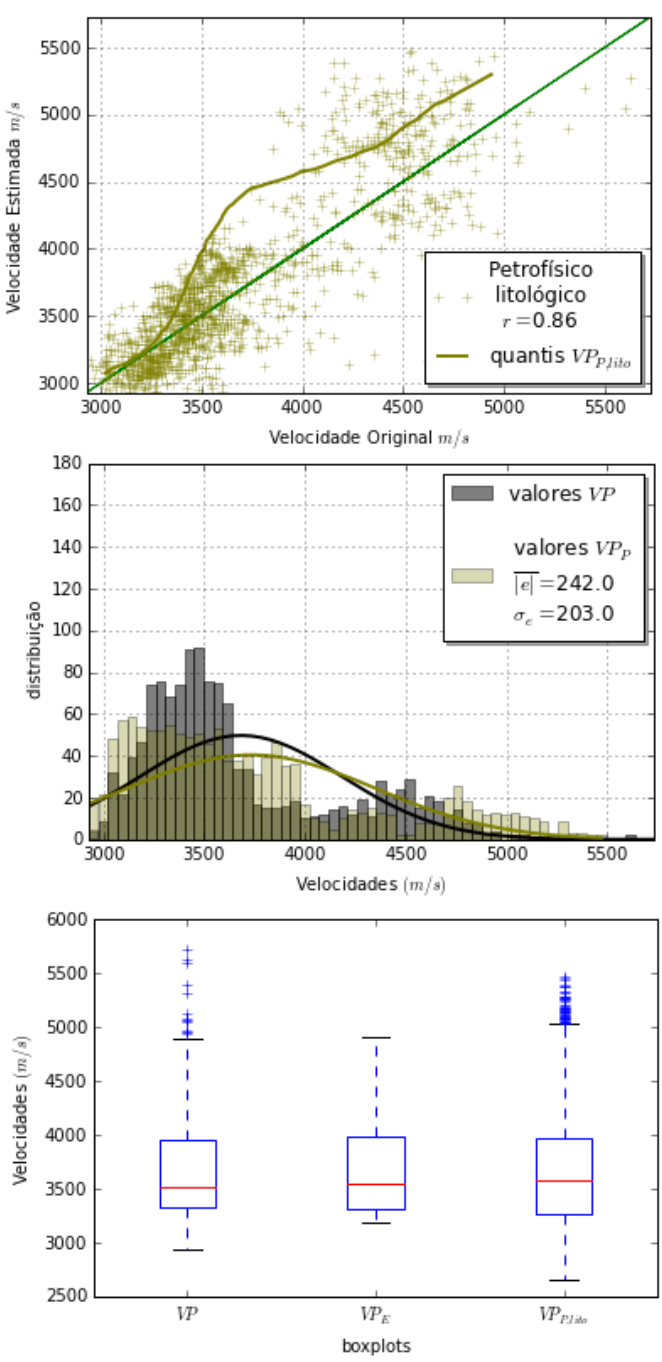

Figura 4 - Gráficos de dispersão, histogramas e diagramas de caixa do modelo petrofísico $\left(V P_{p, m}\right)$ para o poço NA-04

É importante salientar que o modelo petrofísico apresenta resultados coerentes sem a necessidade de realizar uma calibração de constantes, portanto, é completamente independente do perfil sônico. A análise dos mapas, permite verificar a influência destes dados no contexto regional da bacia, e como as tendências dos modelos também ocorrem nos dados interpolados. Na figura 5, por exemplo, temos os mapas de impedância acústica na profundidade de $3038 \mathrm{~m}$, onde os valores desta propriedade se mostram mais homogeneizados no mapa originado dos modelos petrofísicos. Tendência também que pode ser observada nos mapas de refletividade (Figura 5) e amplitude sísmica, ambos na profundidade de $3038 \mathrm{~m}$. Os resultados mostram que o modelo petrofísico é um modelo extremamente versátil, podendo substituir o perfil sônico, no caso da completa inexistência da mesma, e produzir resultados coerentes com os valores de velocidade do poço. A calibração do modelo com o perfil sônico e com a litologia pode, inclusive, produzir melhores resultados. Os mapas obtidos demonstram a tendência dos modelos empíricos, de produzir valores próximos a uma média, influencia também a interpolação destes valores, produzindo mapas que não evidenciam pontos particulares em subsuperfície.

\section{Discussão e Conclusões}

A principal vantagem do modelo petrofísico em relação aos modelos empíricos, é que sua equação está embasada em fundamentos teóricos, portanto, os erros encontrados estão relacionados aos valores das constantes ou das variáveis. Ao aplicar as estimativas para as constantes $\rho_{\text {ma }}$ e $\Delta_{\text {ma }}$ no modelo petrofísico, estamos compensando os erros de outras unidades como por exemplo o valor de $\mathrm{V}_{\mathrm{i}, \mathrm{SH}}$ que, neste poço, possui forte influência dos feldspatos contidos nos arcóseos.

A análise dos resultados, efetuada também nos demais poços, demonstra que o modelo empírico possui melhores resultados pois este apresenta resultados próximo a uma média, o que é justificado pelos valores altos centrais no histograma, e pela curva gaussiana cujo ajuste superior ultrapassa a curva original do poço. Já o modelo petrofísico ele apresenta uma distribuição mais ampla de valores, se mantendo (diversas vezes) simétrico à lineação central do gráfico de dispersão (situação observada também nos histogramas), o que significa que o modelo procura se ajustar para valores altos (e incomuns até) de velocidades.

Estes comportamentos se refletem nos mapas gerados onde, os mapas derivados do modelo empírico tende a homogeneizar mais os valores, enquanto que os mapas oriundos do modelo petrofísico tendem a evidenciar mais as anomalias encontradas.

\section{Agradecimentos}

Agradecemos ao PIBIC-ON/CNPq-MCT pelo apoio no desenvolvimento deste trabalho.

\section{Referências}

Augusto, F. O. A., 2009. Mapas de Amplitude Sísmica para Incidência Normal no Reservatório Namorado, Bacia de Campos. Ph. D. Thesis, Observatório Nacional (ON), Rio de Janeiro (RJ), Brasil.

Gardner, G. H. F., Gardner, L. W., and Gregory, A. R., 1974, Formation Velocity and Density - The Diagnostic Basis for Stratigraphic traps, Geophysics, 39: 770-780.

Isaaks, E. H., Srivastava, R. M., 1989. Applied Geostatistics., oxford university press, New York, USA.

Magalhães F. M., Martins J. L., 2012. Petrophysical Model for Bulk Density of Complex Lithologies, Revista Brasileira de Geofísica, 30(1): 1-17

Sarma, D. D., 2009. Geostatistics with Applications in Earth Sciences. 1 ed., Springer, New York, USA. 
Siqueira, T. B., Martins, J. L., 2011. Um Modelo Petrofísico para Estimativa de Vagarosidades Sônicas, Proceedings 12th International Congress of The Brazilian Geophysical Society, Rio de Janeiro (RJ), Brazil, p.1-4,

Yilmaz, O., 1987. Seismic Data Processing, Society of Exploration of Geophysics- Investigations in Geophysics, vol. 2. 

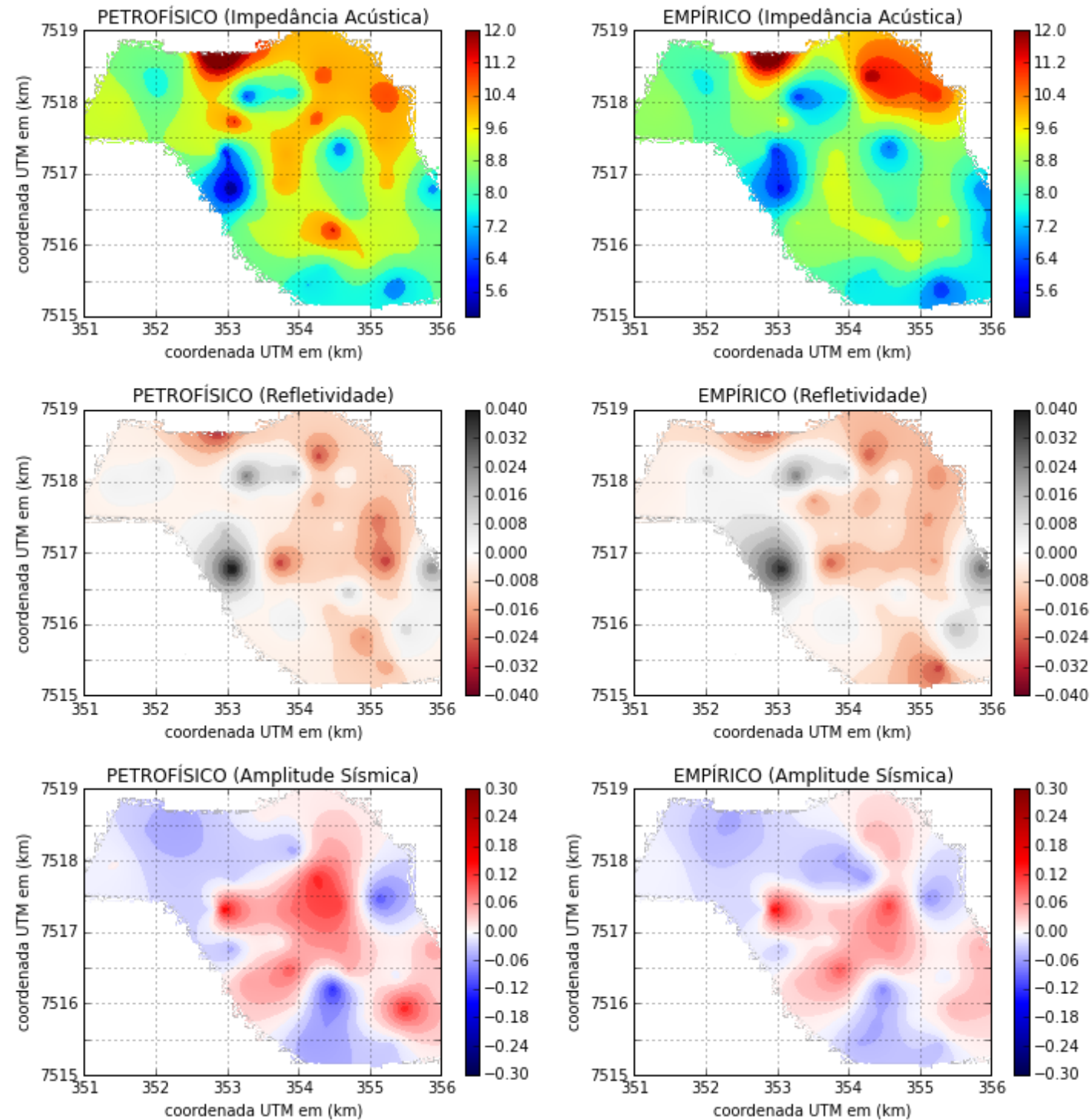

Figura 5 - Mapas de impedância acústica, refletividade e amplitude sísmica no reservatório namorado, para a profundidade de 3038 metros. 